\title{
Observation of Comet C/1999 S4 (LINEAR)
}

\author{
C.Y. Lin and W.H. Ip \\ Graduate Institute of Astronomy, National Central University, 32054 \\ Chung-Li, Taiwan
}

\begin{abstract}
Comet C/1999 S4 (LINEAR) with a maximum magnitude of $6 \mathrm{mag}$ was the brightest comet in 2000. The 24" telescope on NCU campus was used to observe its coma behavior in June and July of 2000 as it approached perihelion. The observations were carried out using the comet filters designed by the European Space Agency for the groundbased observational program of Comet Wirtanen (target comet of the Rosetta mission). Comet C/1999 S4 (LINEAR) ended up disrupting into many small fragments near perihelion. We are in the process of analyzing the time variations of the cometary dust coma and dust tail to examine the physical condition prior to the destruction of the nucleus of this intriguing comet. An image processing method will be developed to trace small-scale structures in the dust tail.
\end{abstract}

\section{Observation in Taiwan}

Comet C/1999 S4 (LINEAR) was observed in June and July 2000 using the 24" Cassegrain reflector on NCU campus (Fig 1.a - 1.d). Besides the I-band filter, the comet filter set provided by ESA was used to carry out photometric imaging observations. The unexpected breakup of this bright comet near perihelion between July 6 and July 24 has attracted wide-ranging interest. This is because the dynamical behavior of the dust tail and the gas coma carries a wealth of important information on the composition and internal structure of the cometary nucleus. The present work serves as a pilot project for our participation in a world-wide network of cometary research.

\section{Data Analysis}

Spectroscopic observations indicated that the CN emission was deficient in this comet (Schleicher 2000). For this reason, we have not been able to obtain a good-quality coma image in $\mathrm{CN}$ emission. We have, however, made a series of narrow-band images of Comet C/1999 S4 (LINEAR) in $\mathrm{C}_{2}$ emission. We are in the process of making careful subtraction of the continuum emission representing the dust component. A ring-mask image processing technique has also been developed to enhance the features of the dust and gas comas (see Fig 2.a and 2.b - kindly provided by Z. W. Zhang). 


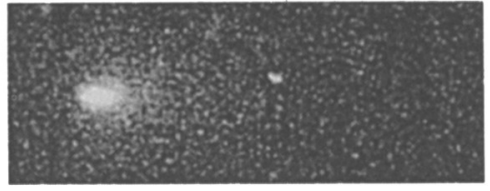

Fig 1. $\mathrm{C}_{2}+$ Continuum (2000/06/23.)

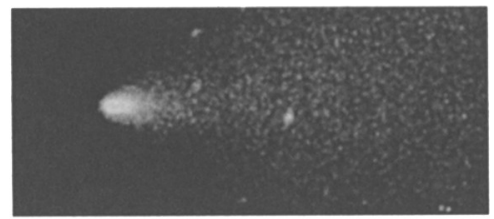

Fig 1.c I-band filter

(2000/06/23.)

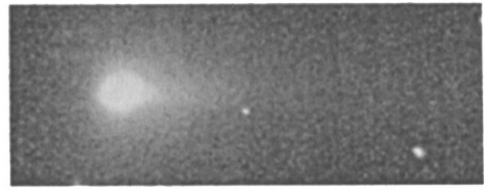

Fig 1.b $\mathrm{C}_{2}+$ Continuum (20000704)

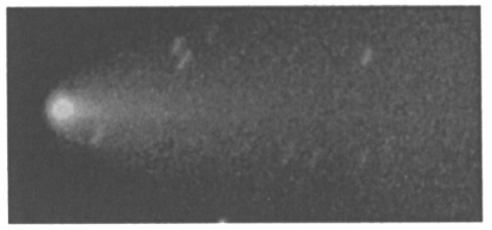

Fig 1.d l-band filter

(2000/07/04.)

Figure 1. Photometric images of Comet C/1999 S4 (LINEAR) taken at NCU observatory on June 23 and July 4, 2000. (a) and (b) $\mathrm{C}_{2}+$ continuum filter images; (c) and (d) I-band filter images.

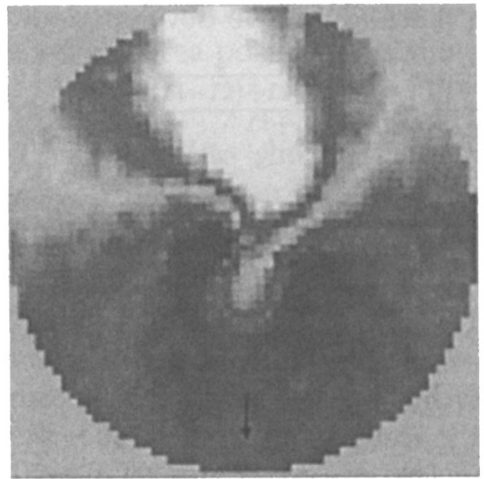

Fig 2.a Ring-masked image I-band

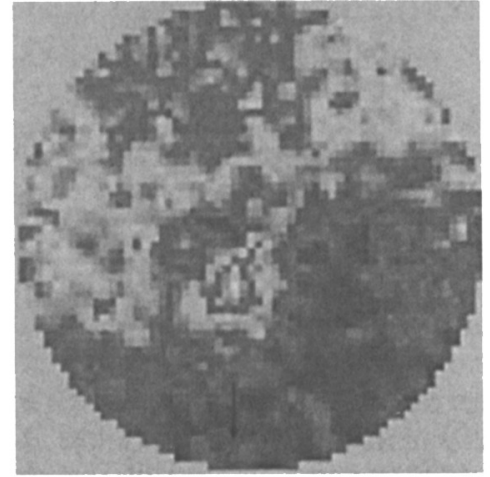

Fig 2.b Ring-masked image $\mathrm{C}_{2}+$ cont.

Figure 2. Ring masked images of (a) Dust continuum and (b) $\mathrm{C}_{2}+$ continuum. The solar direction is indicated by the arrow.

\section{References}

Schleicher, D. G. 2000, IAU Circ, 7342. 